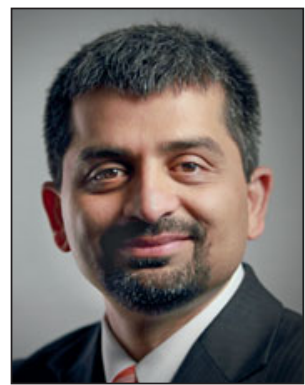

\section{Shekhar Garde}

Guest Editor for this issue of MRS Bulletin

The Howard P. Isermann Department of

Chemical and Biological Engineering,

Rensselaer Polytechnic Institute, USA;

tel. 518-276-6298; and email gardes@rpi.edu.

Garde is the Dean of Engineering and Elaine

S. and Jack S. Parker Chaired Professor at Rensselaer Polytechnic Institute. His work focuses on understanding the role of water in biological structure, function, and interactions using molecular theory and simulations. His awards and honors include the NSF CAREER Award (2001), Vaughan Lectureship at CalTech (2011), and Fellowships of AIMBE (2013) and AAS (2014). He is a co-leader of the Molecularium Project and a co-executive producer of the IMAX movie Molecules to the MAX.

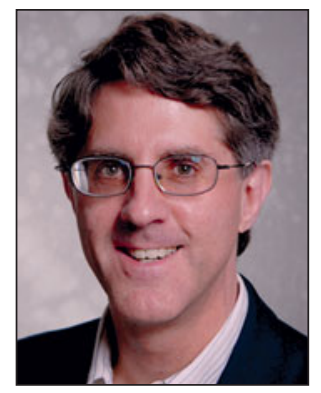

\section{Mark Schlossman}

Guest Editor for this issue of MRS Bulletin

Department of Physics, University of Illinois at Chicago, USA; tel. 312-996-8787; and emailschloss@uic.edu.

Schlossman is a professor in the Department of Physics at the University of Illinois at Chicago. He studies liquid surfaces and interfaces, with a recent emphasis on the role of electrostatics in interfacial phenomena. He is a co-author, along with Peter S. Pershan, of Liquid Surfaces and Interfaces: X-ray Synchrotron Methods, published by Cambridge University Press.

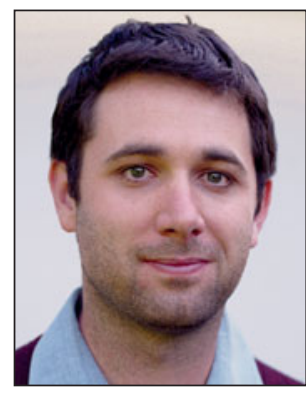

\section{Brendan Allison}

Department of Chemistry and Biochemistry, University of California, Santa Barbara,

USA; email ballison@chem.ucsb.edu.

Allison is a scientific writer at the University of California, Santa Barbara. He holds a bachelor's degree in literary journalism from the University of California, Irvine, with prior publications at the Santa Barbara News Press. He has experience in photovoltaic research and development, and currently works on topics related to water and biomolecular hydration.

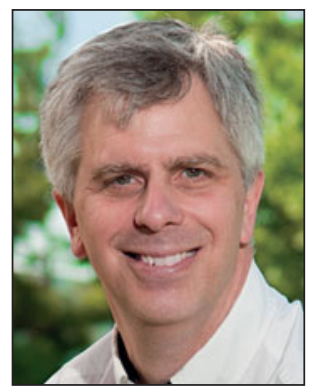

\section{Paul Fenter}

Chemical Sciences and Engineering,

Argonne National Laboratory, USA;

tel.630-252-7053; and email fenter@anl.gov. Fenter is a senior scientist and group leader at Argonne National Laboratory. He has a PhD degree in physics from the University of Pennsylvania. He studies the structure and reactivity of solid-liquid interfaces, ranging from mineralwater interfaces found in geochemical systems to electrified interfaces in energy-storage systems, through the use and development of high resolution $\mathrm{x}$-ray-based scattering. He was awarded the Warren Diffraction Physics Award from the American Crystallographic Association in 2012 and is a Fellow of the American Physical Society.

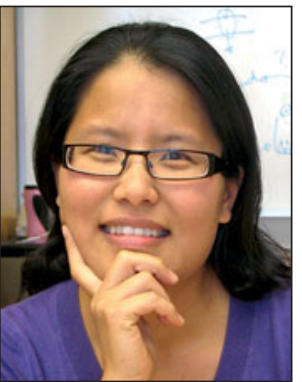

Songi Han

Department of Chemistry and

Biochemistry, University of California,

Santa Barbara, USA; tel. 805-893-4858;

and email songi@chem.ucsb.edu.

Han received her doctoral degree in natural sciences (Dr.rer.nat) from Aachen University of Technology (RWTH), Germany, in 2001. She pursued her postdoctoral studies at the University of California, Berkeley, sponsored by the Feodor Lynen Fellowship of the Alexander von Humboldt Foundation. She joined the faculty at UCSB in 2004, received tenure in 2010 , and was promoted to full professor in 2012. She is a recipient of the 2008 Packard Fellowship, the 2010 Dreyfus-Teacher Scholar Award, and the 2011 NIH Innovator Award.

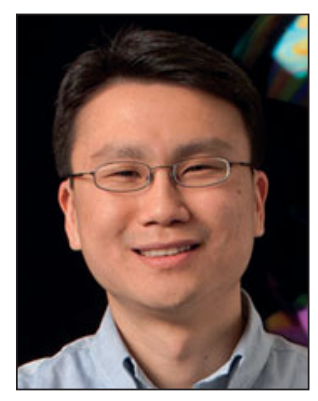

Daeyeon Lee

Department of Chemical and

Biomolecular Engineering, University of Pennsylvania, USA; tel. 215-573-4521; and email daeyeon@seas.upenn.edu.

Lee is an associate professor of chemical and biomolecular engineering at the University of Pennsylvania. He received his BS degree in chemical engineering from Seoul National University in 2001 and his $\mathrm{PhD}$ degree in chemical engineering at MIT in 2007. His research interests include the structure-property relationship of nanoparticle assemblies, interfacial behavior of Janus particles, and microfluidic fabrication of functional structures. His awards include the 2010 Victor K. LaMer Award, an NSF CAREER Award, the 2013 3M Nontenured Faculty Award, the 2013 AIChE NSEF Young Investigator Award, and the 2014 Unilever Award for Young Investigator in Colloid and Surface Science.

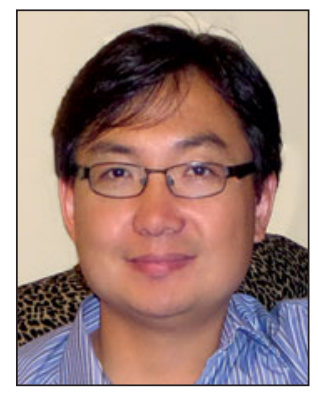

Sang Soo Lee

Chemical Sciences and Engineering,

Argonne National Laboratory, USA;

tel. 630-252-6679; and email sslee@anl.gov. Lee is a geochemist at Argonne National Laboratory. He received his $\mathrm{PhD}$ degree in earth and environmental sciences from the University of Illinois at Chicago in 2007. His current research focuses on in situ and real-time observations of molecular-scale processes at mineral-water interfaces in geochemical systems using synchrotron-based $x$-ray scattering and spectroscopic techniques.

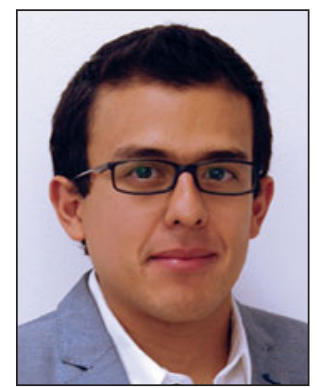

Daniel F. Moyano

Department of Chemistry, University

of Massachusetts Amherst, USA;

emaildmoyano@chem.umass.edu.

Moyano is a PhD candidate in the College of Natural Sciences at the University of Massachusetts Amherst, under the supervision of Vincent $M$. Rotello. In 2009, he received his BSc degree in chemistry at the National University of Colombia. His current research focuses on structure-activity relationship studies of nanoparticle surfaces for immunomodulation. 


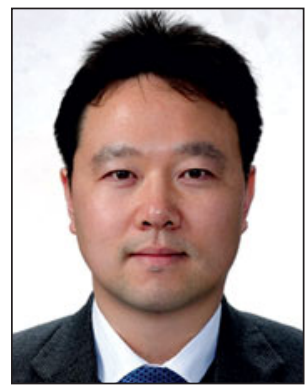

Bum Jun Park

Department of Chemical Engineering, Kyung Hee University, South Korea; tel. +82-31 201-2429; and email bjpark@khu.ac.kr.

Park is an assistant professor in the Department of Chemical Engineering at Kyung Hee University. He received his BS degree in chemical engineering at Hanyang University, South Korea, in 2002 and his PhD degree in chemical engineering at the University of Delaware in 2010. He spent two years as a postdoctoral researcher in chemical and biomolecular engineering at the University of Pennsylvania. His research interests focus on soft matter physics and interfacial engineering.

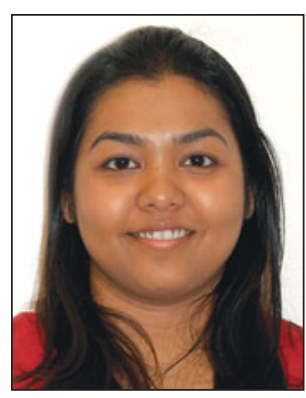

Moumita Ray

Department of Chemistry, University

of Massachusetts Amherst, USA;

email moumita@chem.umass.edu.

Ray is a graduate student in the Department of Chemistry at the University of Massachusetts Amherst, under the guidance of Vincent M. Rotello. She received her BSc and MSc degrees in chemistry from Presidency College, India. Her current research involves understanding nanoparticle-protein interaction and intracellular protein delivery.

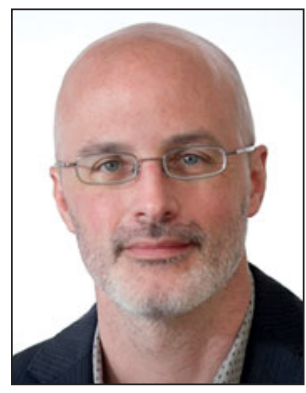

Vincent M. Rotello

Department of Chemistry, University

of Massachusetts Amherst, USA;

email rotello@chem.umass.edu.

Rotello is the Goessmann Professor and University Distinguished Professor of Chemistry at the University of Massachusetts at Amherst. He received the NSF CAREER, Cottrell Scholar, and Camille Dreyfus Teacher-Scholar Awards; the Sloan Fellowship; and the Langmuir Lectureship. $\mathrm{He}$ is a Fellow of the AAAS and of the Royal Society of Chemistry (UK). He is Editor in Chief of Bioconjugate Chemistry and Editorial Board member of 12 other journals. His research program focuses on engineering the interface between hard and soft materials for nanotechnology and nanomedicine, with more than 400 papers published.

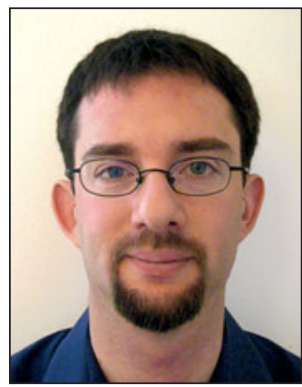

Benjamin Rotenberg

CNRS and Sorbonne Universités,

Université Pierre et Marie Curie, France;

tel. +33 (0)1442722 03; and

email benjamin.rotenberg@upmc.fr.

Rotenberg is a CNRS researcher at Université Pierre et Marie Curie. He graduated from the École Normale Supérieure and received his PhD degree in physical chemistry at UPMC in 2007. He spent his postdoctoral period at the AMOLF Institute, The Netherlands. His research focuses on multiscale modeling of charged porous materials in energy and the environment. In 2013, he received the Michel Gouilloud-Schlumberger Prize from the French Academy of Sciences and the Young Researcher Award from the joint division of the French Chemical and Physical Societies.

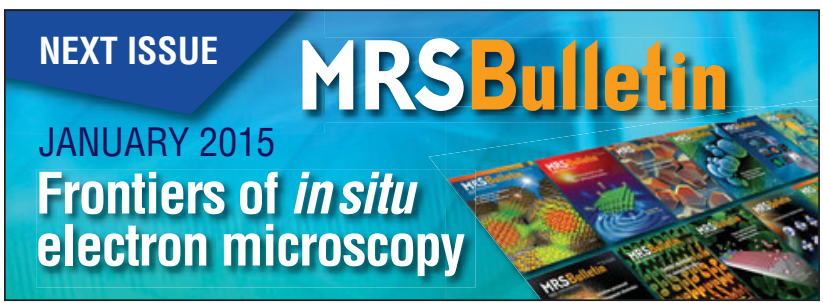

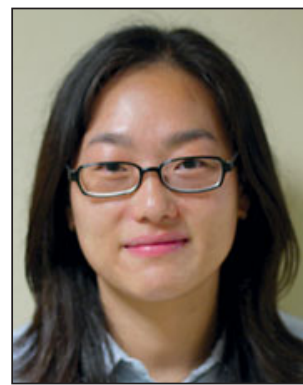

Jinsuk Song

Department of Chemistry and Biochemistry, University of California, Santa Barbara, USA email jsong@chem.ucsb.edu.

Song received her doctoral degree in physics from the Korea Advanced Institute of Science and Technology in 2010 while studying water and ion interaction. She completed her postdoctoral studies at Lehigh University and the University of California, Los Angeles. She is currently a postdoctoral scholar at the University of California, Santa Barbara, and is interested in biomolecular interactions and hydration. She was a recipient of the KBS Science/Engineering Scholarship from 2005 to 2006.

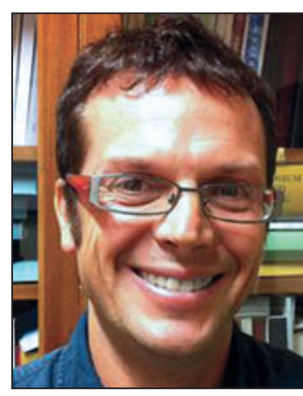

Alberto Striolo

Department of Chemical Engineering,

University College London, United

Kingdom; tel. 44 (0)20 7679 3826;

and email a.striolo@ucl.ac.uk.

Striolo is a professor of molecular thermodynamics at University College London. He holds $\mathrm{BS}$ and $\mathrm{PhD}$ degrees from the Università degli Studi di Padova, Italy. In 2013, he was named the Lloyd and Joyce Austin Presidential Associate Professor at the University of Oklahoma. He received the Regents' Award for Superior Research and Creative Activity in 2011 from the University of Oklahoma and the HP Outstanding Junior Faculty Award from the ACS. He has co-authored over 100 papers on interfacial aqueous systems, energy storage, and Pickering emulsions.

\section{Pilot Plant Pumps \\ Valveless, Ceramic METERING PUMPS}

\section{Pilot Plants, Test Stands, \& Modular Lab Systems}

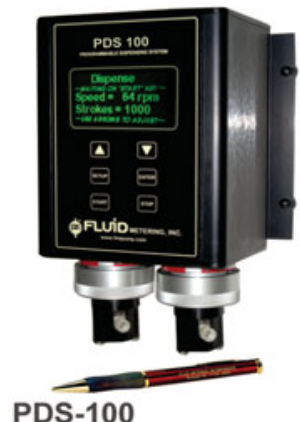

Programmable Dispense System (shown above) $C \epsilon$

\author{
Providing Pilot Plant \\ Fluid Control Solutions \\ for Over 50 Years!
}

- Chemical Pilot Plant

- Fuel Cell Test Stands

- Water Treatment

- Mining Ore Extraction

- Pharmaceutical Pkg.

- Filtration Test Stands

- Environmental Monitoring

- Alternative Energy R\&D

ISO9001

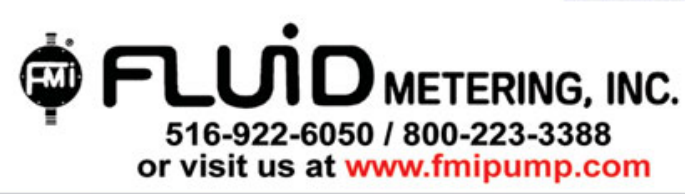

